\title{
Long non-coding RNA ENST00000547547 inhibits cell proliferation, invasion and migration in colorectal cancer cells
}

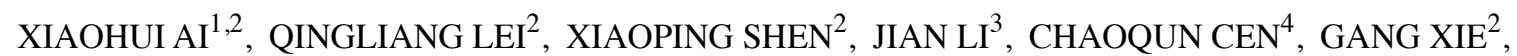 \\ BO LUO ${ }^{2}$, WENSHUO ZHU ${ }^{2}$, GUI HU ${ }^{1}$, HAO WU ${ }^{1}$, MIAO CHEN ${ }^{1}$, \\ XIAORONG LI ${ }^{1}$ and CHANGWEI LIN ${ }^{1}$ \\ ${ }^{1}$ Department of Gastrointestinal Surgery, The Third Xiangya Hospital of Central South University, Changsha, \\ Hunan 410013; ${ }^{2}$ Department of Tumor Surgery, Shaoyang Hospital of TCM, Shaoyang, Hunan 422000; \\ Departments of ${ }^{3}$ Nuclear Medicine and ${ }^{4}$ Emergency Medicine and Intensive Care Unit, \\ The Third Xiangya Hospital of Central South University, \\ Changsha, Hunan 410013, P.R. China
}

Received August 25, 2017; Accepted August 10, 2018

DOI: $10.3892 /$ or.2018.6834

\begin{abstract}
Colorectal cancer (CRC) is one of the most common and lethal types of cancer worldwide. Multiple lines of evidence have illustrated that long non-coding RNAs (lncRNAs) are critical molecules in the regulation of CRC development and progression. The identification of new lncRNAs is expected to provide new biomarkers and potential therapeutic targets for CRC diagnosis and prevention. Previous research has revealed that, ENST00000547547, a 434-bp 1ncRNA on human chromosome12q15 (RP11-611E13.3-001), was found to be downregulated in CRC tissues from lncRNA microarray studies. However, its role in the development and progression of CRC remains largely unknown. In the present study, we demonstrated that the ENST00000547547 level is significantly downregulated in CRC tissues compared to normal tissues. Furthermore, the overexpression of ENST00000547547 inhibited the cell proliferation, invasion and migration of CRC cells in vitro and decreased tumorigenesis ability in vivo. Furthermore, we provided the first evidence that ENST00000547547 inhibited the proliferation of CRC cells by
\end{abstract}

Correspondence to: Professor Xiaorong Li or Dr Changwei Lin, Department of Gastrointestinal Surgery, The Third Xiangya Hospital of Central South University, Tongzipo Road, Changsha, Hunan 410013, P.R. China

E-mail: lixiaorong@medmail.com.cn

E-mail: linchangwei1987@csu.edu.cn

Abbreviations: mock, pcDNA3.1 plasmid transfected group or Lv-NC stable cells; ENST547, pcDNA3.1-ENST00000547547 plasmid transfected group or Lv-ENST00000547547 stable cells

Key words: long non-coding RNA, ENST00000547547, colorectal cancer, apoptosis, epithelial mesenchymal transition affecting the cell cycle and apoptosis. Finally, we demonstrated that the epithelial-mesenchymal transition (EMT) process was associated with the inhibitory effects of ENST00000547547 on the invasion and migration of CRC cells. In summary, the present study indicated that lncRNA ENST00000547547 acted as a tumor suppressor in CRC, which may provide us with a new biomarker for CRC prognosis and a potential therapeutic target for molecular cancer therapy.

\section{Introduction}

Colorectal cancer (CRC) is the third most common type of cancer worldwide (1). Due to its rapid progression, invasion and metastasis, CRC is one of the leading causes of cancer-related deaths globally (2). In China, the newly diagnosed cases with CRC in 2011 were 310,244, accounting for $9.20 \%$ of the overall new cancer cases (3). Although treatment for CRC has been developed, the survival rate of CRC patients remains poor (4). Thus, a deeper understanding of the molecular and genetic networks in CRC development and progression and the identification of new molecular markers or factors are urgent.

Long non-coding RNAs (lncRNAs), generally longer than 200 nucleotides in length, are widely produced in humans and they are emerging as important regulators in a wide range of biological processes, such as proliferation, apoptosis, cell cycle arrest, cell migration and invasion (5). In recent years, lncRNAs such as ANRIL, GAS5, UCA1 and HULC were identified as functional factors in tumor initiation and progression (6-9). In human CRC, hundreds of novel lncRNAs with dysregulated expression were found by a genome-wide study and some of them demonstrated co-regulated expression patterns with their neighboring protein-coding genes, indicating that they displayed enhancer-like functions (10). In further clinical and functional studies, several lncRNAs were found to be important regulators of tumorigenesis in CRC, including PRNCR1, MALAT-1, HOTAIR and AK027294, which partly or totally affected cancer cell proliferation, metastasis, cell cycle 
progression, apoptosis and epithelial-mesenchymal transition (EMT) (11-14).

LncRNA ENST00000547547 is a 434-bp transcript on human chromosome12q15 (RP11-611E13.3-001) that had a distinct expression pattern in our previous lncRNA microarray assay (15). However, the potential role of ENST00000547547 in the development and progression of CRC remains unknown. In the present study, we have assessed the expression of ENST00000547547 in CRC tissues and cells. A functional overexpression model was used to investigate its function in the proliferation, invasion and migration of CRC cells in vitro and tumorigenesis abilities in vivo. Furthermore, we investigated the potential functional mechanism of ENST00000547547 in regulating $\mathrm{CRC}$ cell proliferation, invasion and migration.

\section{Materials and methods}

Tissue collection. CRC tissues and adjacent non-cancerous tissues were collected from 21 patients who had undergone surgical resection of CRC at the Cancer Hospital of Hunan from January to September of 2015. The normal tissue samples were $5 \mathrm{~cm}$ from the edge of the tumor and were identified by a pathologist. Prior to the surgical resections, no preoperative treatment had been administered to these patients. All tissue samples were immediately frozen in liquid nitrogen after being surgically resected and then stored at $-80^{\circ} \mathrm{C}$ until required for the analyses. Prior to sample collection, written informed consent was obtained from all patients, and all of the experiments were approved by the Research Ethics Committee of the Shangyao Hospital of TCM.

Cell lines and culture conditions. Human normal colorectal cell line NCM460 and CRC cell lines COLO320, HCT116 and LoVo were purchased from Auragene Bioscience Co. (Changsha, China). NCM460 cells were cultured in McCoy's 5A medium (Thermo Fisher Scientific, Inc., Waltham, MA, USA) supplemented with $10 \%$ fetal bovine serum (FBS; Gibco; Thermo Fisher Scientific, Inc.). COLO320, HCT116 and LoVo cells were cultured in Dulbecco's modified Eagle's medium (DMEM; Gibco; Thermo Fisher Scientific, Inc.). All cells were cultured at $37^{\circ} \mathrm{C}$ in humidified air with $5 \% \mathrm{CO}_{2}$. All media were supplemented with $10 \%$ FBS, $100 \mathrm{U} / \mathrm{ml}$ penicillin and $100 \mathrm{mg} / \mathrm{ml}$ streptomycin (Invitrogen, Shanghai, China).

RNA extraction and $q R T-P C R$ analyses. Total RNA was extracted from tissues or cultured cells with TRIzol reagent (Invitrogen, Shanghai, China). For quantitative RT-PCR (qRT-PCR), cDNA was synthesized using random primers and a reverse transcription kit (Takara Biotechnology Co., Ltd., Dalian, China). qRT-PCR analyses were performed using SYBR-Green qPCR Mix (Toyobo Life Science, Osaka, Japan). All protocols were performed according to the manufacturer's instructions. The qRT-PCR assays and data collection were performed using an ABI 7300 instrument. Primers for qRT-PCR were synthesized by Invitrogen (Shanghai, China) and the sequences were as follows: ENST00000547547 sense, 5'-TTTTCTAAGGCACCAACT-3' and antisense, 5'-CCA AATGCTCTAAGGGA-3'; ZEB1 sense, 5'-AAATGGAAC ACCAGATGC-3' and antisense, 5'-TTACACCCAGACTGC GTC-3'; Snal sense, 5'-GCCTGTCTGCGTGGGTTT-3' and antisense, 5'-GTGAGTCTGTCAGCCTTTGTCC-3'; vimentin sense, 5'-CCTGAACCTGAGGGAAACTAATC-3' and antisense, 5'-GAAGTTTCGTTGATAACCTGTCC-3'; E-cadherin sense, 5'-AGGTCTCCTCTTGGCTCTG-3' and antisense, 5'-AATAGGCTGTCCTTTGTCG-3'; N-cadherin sense, 5'-GCATCATCATCCTGCTTATCC-3' and antisense, 5'-GTCCTGGTCTTCTTCTCCTCC-3'; $\beta$-actin sense, 5'-AGG GGCCGGACTCGTCATACT-3' and antisense, 5'-GGCGGC ACCACCATGTACCCT-3'. Thermocycling conditions were as follows: Initial DNA heat denaturation at $95^{\circ} \mathrm{C}$ for $3 \mathrm{~min}$, followed by 39 cycles at $95^{\circ} \mathrm{C}$ for $10 \mathrm{sec}$ and then, annealing and extending at $60^{\circ} \mathrm{C}$ for $30 \mathrm{sec}$ in the qRT-qPCR experiment. The expression levels of the target genes were normalized to the transcription level of $\beta$-actin. Each sample was analyzed in triplicate.

In situ hybridization (ISH) analysis. Paraffin-embedded sections of CRC and adjacent non-cancerous tissues were used to detect ENST00000547547 expression using the RNAscope ${ }^{\circledR}$ Assay-Brown (Advanced Cell Diagnostics, Advanced Cell Diagnostics, Inc., Newark, CA, USA) according to the manufacturer's instructions with the following modifications: Antigen retrieval for $15 \mathrm{~min}$, protease digestion for $30 \mathrm{~min}$ at $37^{\circ} \mathrm{C}$, probe incubation at $42^{\circ} \mathrm{C}$ overnight. The probe sequence for ENST00000547547 was 5'-GGTTTACTCTTCCCCTCT GTTC-3'. The expression level of ENST00000547547 in the nucleus was visualized with DAB and quantitated using an automatic imaging system (Leica DMLA; Leica Microsystems, Wetzlar, Germany).

Cell transfection. For the overexpression of ENST00000547547, a pCDNA3.1-ENST00000547547 plasmid was constructed by Auragene Bioscience Co. The plasmid carrying pCDNA3.1-ENST00000547547 $(1 \mu \mathrm{g} / \mu \mathrm{l})$ was transfected into the CRC cell lines HCT116 and LoVo using Lipofectamine 2000 (Invitrogen; Thermo Fisher Scientific, Inc.) at a Lipofectamine 2000:pCDNA3.1-ENST00000547547 ratio of 1:2.

Cell proliferation assay. After pCDNA3.1-ENST00000547547 transfection, the transfected and control cells were seeded into 96-well plates at an initial density of 5,000 cells/well. After culture for 24,48 and $72 \mathrm{~h}$, the cells were treated with $10 \mu 1$ 3-(4,5-dimethylthiazol-2-yl)-2,5-diphenyltetrazolium bromide (MTT) solution (Sangon Biotech, Co., Ltd., Shanghai, China) by adding it into each well. The cells were incubated at $37^{\circ} \mathrm{C}$ for another $4 \mathrm{~h}$, and then the medium was carefully removed and $150 \mu \mathrm{l}$ dimethyl sulfoxide (DMSO) solution (MP Biomedicals, Santa Ana, CA, USA) was added to lyse the cells for $10 \mathrm{~min}$. Finally, the absorbance was assessed at $570 \mathrm{~nm}$ using a Multiskan MK microplate reader (Thermo Fisher Scientific, Inc., Waltham, MA, USA). All experiments were performed in triplicate.

Tumor formation assay in a nude mouse model. Six 4-week-old male BALB/c nude mice (original weight, $25 \mathrm{~g}$ ) were obtained from the Hunan Branch of the Shanghai Laboratory Animal Center of the Chinese Academy of Sciences (Changsha, China). The mice were housed on a $12 \mathrm{~h} \mathrm{light/dark} \mathrm{cycle}$ under pathogen-free conditions and were fed an autoclaved diet ad libitum. To assess tumor formation, the mice were 
subcutaneously injected with $5 \times 10^{6} / \mathrm{ml}$ HCT116 cells stably expressing Lv-NC or Lv-ENST00000547547. For the tumor formation assay, the tumor volume was calculated as follows: Tumor volume $=\left(\right.$ width $^{2} \mathrm{x}$ length $) / 2$. At 28 days after the Lv-NC and Lv-ENST00000547547 inoculation, the mice were sacrificed and the tumors derived from each group were used for comparisons. The protocol was approved by the Ethics Committee on the Animal Experiments of the Third Xiangya Hospital of Central South University.

Cell migration and invasion assays. Cell migration and invasion assays were performed using Transwell chambers (micropore size, $8 \mu \mathrm{m}, 24$-well; Corning ${ }^{\circledR}$; Thermo Fisher Scientific, Inc.) without Matrigel (for migration assays) or with Matrigel (for invasion assays). Both assays were performed according to the manufacturer's instructions. Briefly, the treated cells were plated in the upper chamber at a concentration of $5 \times 10^{4}$ in $500 \mu \mathrm{l} \mathrm{FBS}$-free media. The bottom chamber contained $500 \mu \mathrm{l}$ media with $10 \%$ FBS. The plates were incubated for $24 \mathrm{~h}$, and then the chamber was fixed and stained with crystal violet staining solution (Beyotime Institute of Biotechnology, Haimen, China). The stained cells were imaged under a microscope at $\times 100$ magnification and the optical density (OD) values were detected using a microplate reader (Thermo Multiskan MK3; Thermo Fisher Scientific, Inc.).

Flow cytometric analysis. The CRC cell lines HCT116 and LoVo were transfected with pCDNA3.1-ENST00000547547 or pCDNA3.1-NC for $48 \mathrm{~h}$ and harvested. Then, the cells were washed three times with cold phosphate-buffered saline (PBS) and fixed with cold $70 \%$ ethanol overnight. For the cell cycle analysis, the cells were stained with propidium iodide (PI) (Keygentec, Inc., Nanjing, China) for $30 \mathrm{~min}$ at $4^{\circ} \mathrm{C}$ in the dark. The cell cycle profiles were assayed using flow cytometry (BD Biosciences, Franklin Lakes, NJ, USA). For the apoptosis analysis, the cells were harvested $48 \mathrm{~h}$ after transfection and were stained with Annexin V-fluorescein isothiocyanate (FITC) and PI (Keygentec, Inc.) for $10 \mathrm{~min}$ in the dark at room temperature. The cells were then examined by flow cytometry (BD Biosciences). All of the samples were assayed in triplicate.

Western blot analysis. Cells were lysed using RIPA lysis buffer (Auragene Bioscience Co.), supplemented with a protease inhibitor cocktail (Roche Diagnostics, Indianapolis, IN, USA) and phenylmethylsulfonyl fluoride (PMSF) (Auragene Bioscience Co.). Equal amounts $(25 \mu \mathrm{g})$ of protein were loaded on a $10-12 \%$ SDS-polyacrylamide separating gel. The proteins were then transferred to a Immobilon-P polyvinylidene difluoride (PVDF) membrane (EMD Millipore, Billerica, MA, USA). The membrane was blocked with $3 \%$ bovine serum albumin-Tris-buffered saline with Tween-20 (BSA-TBST) with gentle shaking at room temperature for $90 \mathrm{~min}$. Then, the membrane was probed with the indicated primary antibodies with gentle shaking at $4^{\circ} \mathrm{C}$ overnight. The membranes were washed with TBST (5x3 min) and incubated with specific secondary antibodies at room temperature for $1 \mathrm{~h}$. A $\beta$-actin antibody (cat. no. LCA01; Auragene, Changsha, China) was used as a control and Bcl-2 (1:1,000; cat. no. AM2211; Abzoom, Changsha, China), Bax (1:500; cat. no. AM2198; Abzoom),
PCNA (1:200; cat. no. AM3547; Abzoom), P21 (1:200; cat. no. B7175; Assay Biotechnology, California's San Francisco Bay Area, CA, USA), cyclin D1 (1:1,000; cat. no. ab134175; Abcam, Dallas, TX, USA), cyclin E1 (1:1,000; cat. no. ab33911; Abcam), P27 (1:200; cat. no. YM0496; ImmunoWay, Changsha, China), ZEB1 (1:500; cat. no. 21544-1-AP; ProteinTech, Wuhan, China), Sna1 (1:500; cat. no. ab82846; Abcam), N-cadherin (1:1,000; cat. no. YM0465; ImmunoWay), vimentin (1:800; cat. no. BM0147; Abzoom) and E-cadherin (1:800; cat. no. BM0530; Abzoom) antibodies were used for each group. The second antibody was goat anti-rabbit IgG (H+L)-HRP (1:18,000; cat. no. SA009; Auragene Bioscience Co.) or goat anti-mouse $\operatorname{IgG}(\mathrm{H}+\mathrm{L})-\mathrm{HRP}(1: 18,000$; cat. no. SA001; Auragene Bioscience Co.) incubation for $1 \mathrm{~h}$ in room temperature.

Statistical analysis. All statistical analyses were performed using SPSS 20.0 (IBM Corp., Armonk, NY, USA). Data are expressed as the mean \pm standard deviation (SD) from at least three independent experiments. Statistical analyses were determined with Student's t-test and AVONA as appropriate. All $\mathrm{P}$-values were two-sided and $\mathrm{P}<0.05$ was considered to indicate a statistically significant result.

\section{Results}

ENST00000547547 is downregulated in CRC. The lncRNA microarray data revealed that lncRNA 3837 was downregulated and lncRNA 1181 was upregulated in CRC tissues. In addition, the ENST00000547547 level was decreased in the microarray data in CRC tissues compared to normal tissues (Fig. 1A). Subsequently, its expression was analyzed by qRT-PCR and in situ hybridization assays in CRC and adjacent non-cancerous tissues. The qRT-PCR results revealed that the expression of ENST00000547547 in CRC tissues was significantly decreased compared to adjacent non-tumor tissues (Fig. 1B). The in situ hybridization assay results revealed that the CRC tissues displayed lower ENST00000547547 staining than the adjacent non-cancerous tissues (Fig. 1C). The ENST00000547547 level in the human CRC cell lines COLO320, HCT116 and LoVo, and the normal human colonic epithelial cell line NCM460 was examined by qRT-PCR. As displayed in Fig. 1D, the expression of ENST00000547547 was significantly decreased in CRC cell lines compared to the expression in normal human colorectal cell lines. These results indicated that ENST00000547547 was significantly downregulated in CRC.

Overexpression of ENST00000547547 inhibits CRC cell proliferation and tumor growth. To investigate the potential biological function of ENST00000547547 in CRC cells, an ENST00000547547-overexpressing plasmid was constructed and used to transfect HCT116 and LoVo cells (16). The overexpression efficiency was verified by qRT-PCR analysis (Fig. 2A).

To determine the effect of ENST00000547547 on CRC cell proliferation, an MTT assay and flow cytometric analysis were performed. The MTT results revealed that the overexpression of ENST00000547547 significantly inhibited cell proliferation in the HCT116 and LoVo cells compared to the control group (Fig. 2B). As displayed in Fig. 2C, the 

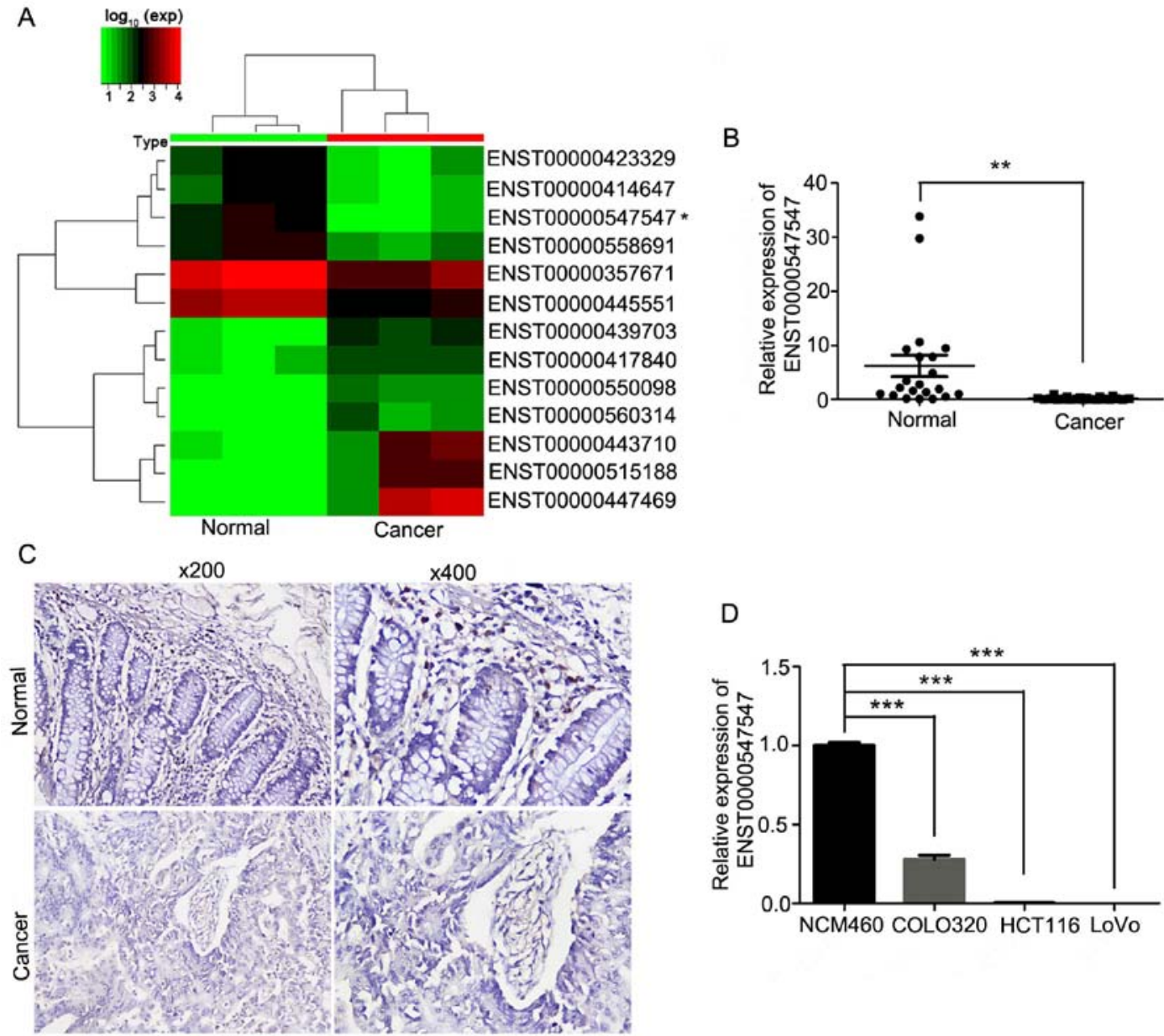

Figure 1. Relative expression of ENST00000547547 in CRC tissues and cells compared with adjacent normal tissues and normal colonic epithelial cells. (A) The IncRNA microarray data revealed that ENST00000547547 expression levels were significantly decreased compared to normal tissues. Expression values are represented in shades of red and green, indicating expression above and below the median expression value, respectively. (B) The relative expression of ENST00000547547 in CRC tissues $(n=21)$ compared with adjacent normal tissue $(n=21)$. The ENST00000547547 expression was examined by qRT-PCR and normalized to the $\beta$-actin expression. The results are presented as the fold-change in tumor tissues relative to normal tissues (shown as $2^{-\Delta \Delta C t}$ ). (C) In the ISH images of paraffin sections of CRC and adjacent normal tissues, ENST00000547547 shows a dot-like nuclear staining pattern with DAB staining. The expression of ENST00000547547 in CRC tissues was lower than in adjacent normal tissues. (D) The ENST00000547547 expression was assessed by qRT-PCR in colorectal cancer cell lines (COLO320, HCT116 and LoVo) and compared with the NCM460 cells; ${ }^{* *} \mathrm{P}<0.01,{ }^{* * * *} \mathrm{P}<0.001$. CRC, colorectal cancer.

overexpression of ENST00000547547 significantly increased cell apoptosis in the HCT116 and LoVo cells. Various mechanisms have been studied to explain lncRNA-mediated cell apoptosis and the most important factor identified is the activation of anti- or pro-apoptotic regulators. Bcl-2 and Bax proteins are one of the many ways to induce apoptosis (17), and proliferating cell nuclear antigen (PCNA) is known as a molecular marker for proliferation $(18,19)$. To confirm the role of ENST00000547547-induced apoptosis, the expression levels of Bcl-2, PCNA and Bax were examined. Western blot analysis revealed that the protein levels of Bax were significantly increased in ENST00000547547-overexpressing cells, whereas the protein levels of Bcl-2 and PCNA were decreased (Fig. 2D). Collectively, these data indicated that the overexpression of ENST00000547547 could inhibit cell growth and promote apoptosis in CRC cells.

To further determine the effect of ENST00000547547 on tumorigenesis, cells stably expressing Lv-NC and Lv-ENST00000547547 were subcutaneously injected into nude mice. Nine days after the injection, a palpable tumor could be observed in both groups. There was a dramatic decrease in tumor volume in the Lv-ENST00000547547 group compared with the Lv-NC group (Fig. 2E). In addition, at 28 days, tumors derived from the Lv-ENST00000547547 group were significantly smaller than those in the control group (Fig. 2F). These results indicated that the overexpression of ENST00000547547 significantly inhibited CRC growth in vitro and in vivo.

Overexpression of ENST00000547547 induces G0/G1 phase arrest in vitro. To further assess whether the antiproliferative effects of ENST00000547547 on CRC cells were mediated by inhibiting cell cycle progression, the cell cycle was evaluated by flow cytometry and western blotting. The flow cytometric results demonstrated that overexpression of ENST00000547547 induced a significant G1-phase increase and caused an obvious reduction in the number of cells in the S phase in the HCT116 and LoVo cells (Fig. 3A and B). Furthermore, western blotting results revealed that the cell cycle-related proteins P21 and P27 were significantly increased in the ENST00000547547-overexpressing cells, 
A

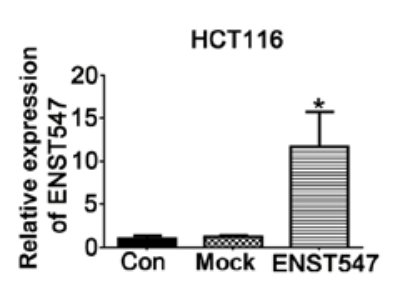

B

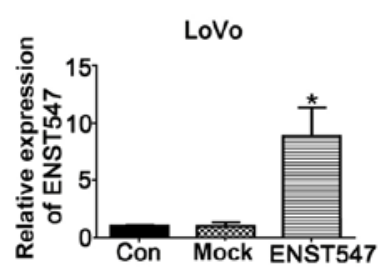

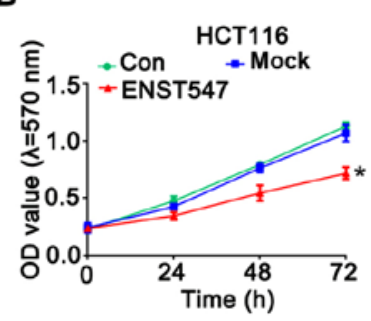

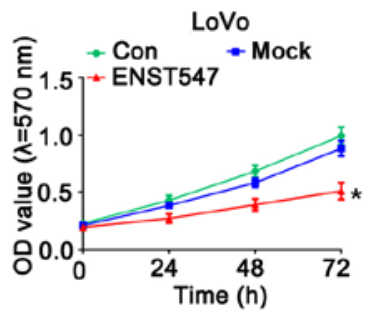

C

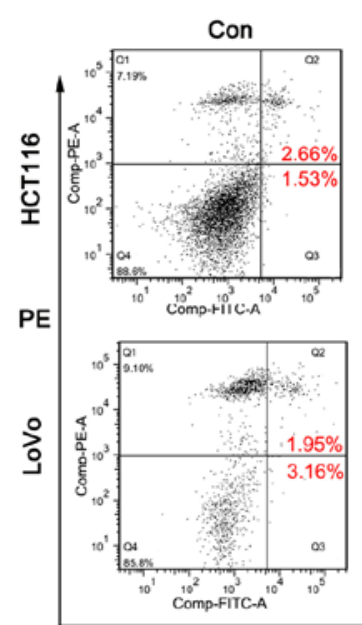

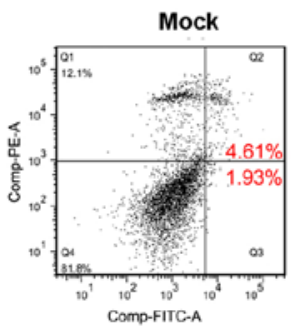
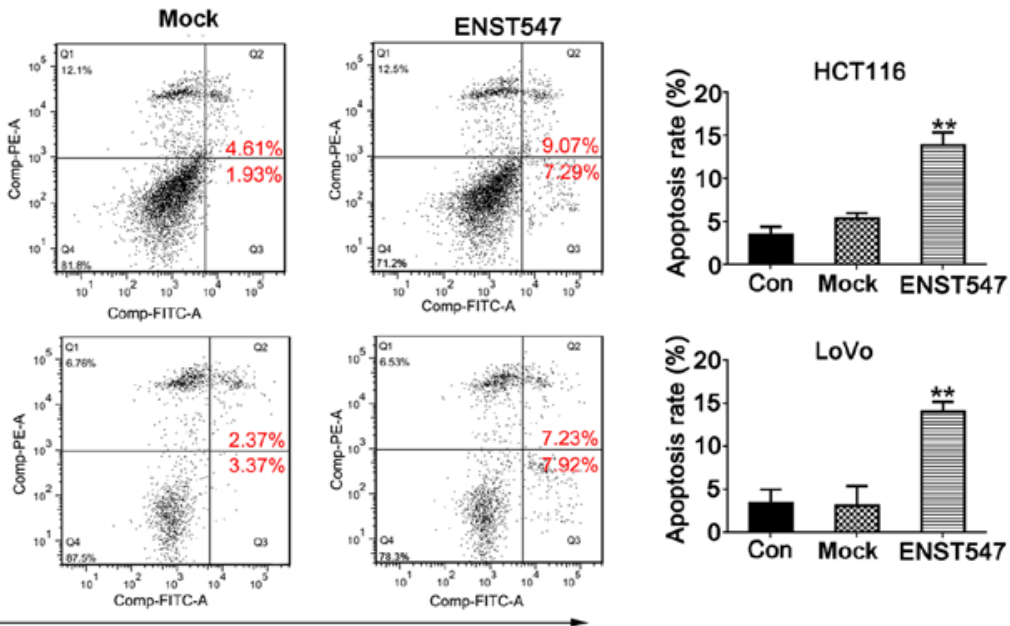

D

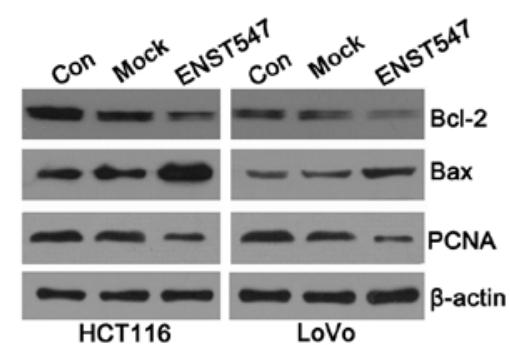

E

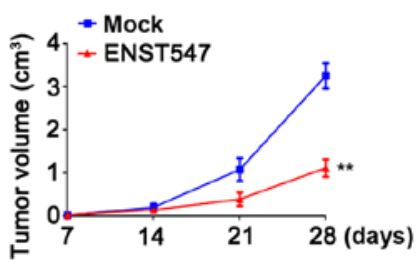

F

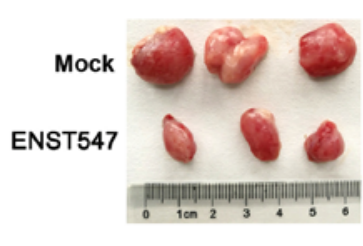

Figure 2. Effects of ENST00000547547 on CRC cell proliferation and tumor growth in vitro and in vivo. (A) The overexpression efficiency of ENST00000547547 was detected by qRT-PCR assay in the HCT116 and LoVo cells which were transfected with pCDNA3.1-ENST00000547547 or pCDNA3.1-NC for 48 $\mathrm{h}$. (B) MTT assays were performed to determine the proliferation of HCT116 and LoVo cells. (C) Flow cytometry with Annexin V/PI staining was used to determine cell apoptosis in HCT116 and LoVo cells. (D) The expression of apoptosis genes (Bcl-2 and Bax) and growth gene PCNA in the HCT116 and LoVo cells was determined by western blot analysis. $\beta$-actin was used as a control. Data represent the mean \pm SD from three independent experiments. (E) Tumor growth curve was based on tumor volumes $(\mathrm{n}=3)$ which were calculated every 7 days after injection of Lv-NC or Lv-ENST00000547547 stable cells of HCT116. (F) The tumors after removal from the mice, at 28 days; ${ }^{*} \mathrm{P}<0.05,{ }^{* *} \mathrm{P}<0.01$. CRC, colorectal cancer; mock, pcDNA3.1 plasmid transfected group or Lv-NC stable cells; ENST547, pcDNA3.1-ENST00000547547 plasmid transfected group or Lv-ENST00000547547 stable cells.

whereas the protein levels of cyclin D1 and E1 were decreased in the HCT116 and LoVo cells (Fig. 3C). These observations indicated that ENST00000547547 may induce cell cycle arrest by affecting the expression levels of cyclins. Collectively, these data demonstrated that ENST00000547547 induced G0/G1 phase arrest in CRC cells.

Overexpression of ENST00000547547 inhibits CRC cell invasion and migration in vitro. To analyze the role of ENST00000547547 in cell invasion and migration, Transwell assays were performed in HCT116 and LoVo cells and the results revealed that ENST00000547547 overexpression greatly decreased cell invasion and migration compared with the control groups (Fig. 4). These data demonstrated that the overexpression of ENST00000547547 inhibited the invasion and migration of CRC cells in vitro.
Overexpression of ENST00000547547 suppresses EMTinducing gene expression. To investigate whether the expression of ENST00000547547 regulated the progress of the epithelial-mesenchymal transition (EMT), we examined the expression levels of ZEB1, Sna1, N-cadherin, vimentin and E-cadherin in ENST00000547547-overexpressing HCT116 and LoVo cells. qRT-PCR analysis indicated that overexpression of ENST00000547547 resulted in an obvious downregulation in the transcription levels of EMT-inducing genes (ZEB1, Sna1) and the biomarkers of mesenchymal cells (N-cadherin and vimentin), as well as a clear upregulation in the transcription level of E-cadherin, which is a hallmark of epithelial cells (Fig. 5A). These results were reconfirmed by western blot analysis (Fig. 5B). These data indicated that ENST00000547547 inhibited the metastasis of CRC cells by suppressing the expression of EMT-inducing genes. 

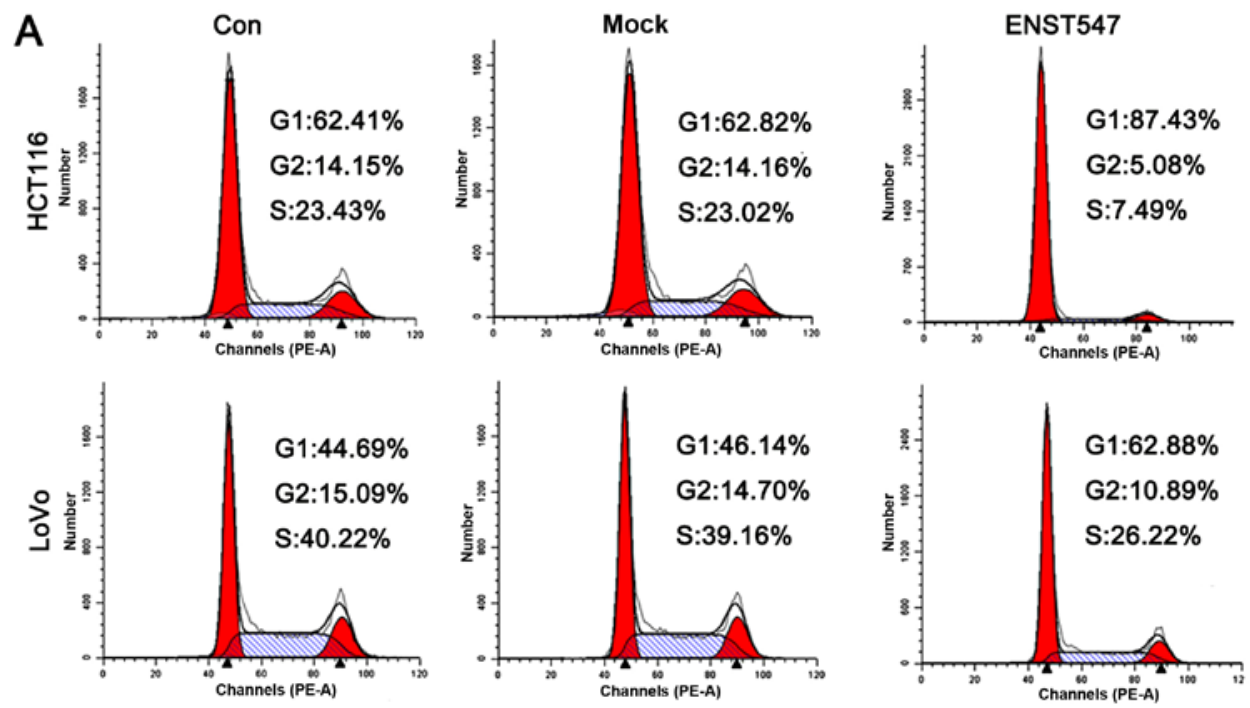

B
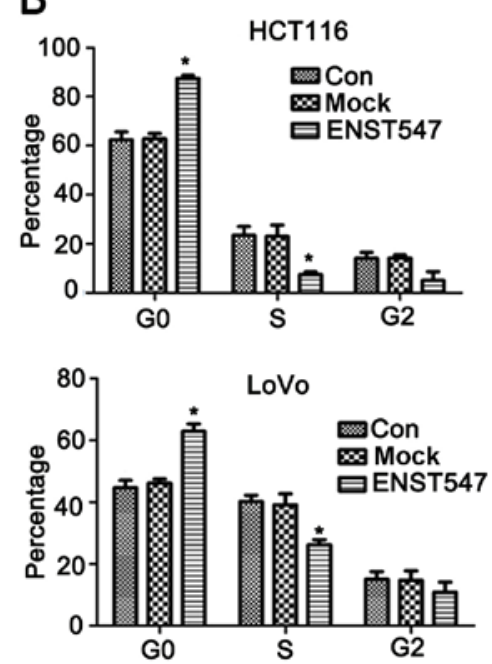

C

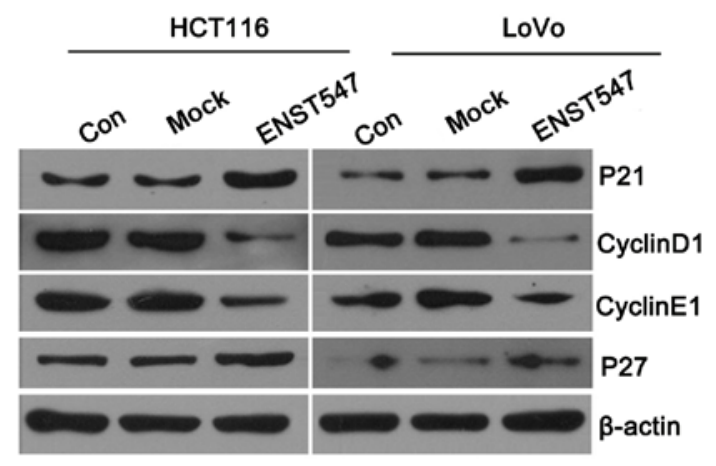

Figure 3. Effects of ENST00000547547 on colorectal cancer (CRC) cell cycle. (A and B) The percentages of HCT116 and LoVo cells in each phase were determined by flow cytometry after the upregulation of ENST00000547547. (C) The protein levels of P21, cyclin D1, cyclin E1 and P27 were determined by western blot analysis in the in HCT116 and LoVo ENST00000547547-overexpressing cells. $\beta$-actin was used as a control. Data represent the mean \pm SD from three independent experiments; "P<0.05. CRC, colorectal cancer; mock, pcDNA3.1 plasmid transfected group or Lv-NC stable cells; ENST547, pcDNA3.1-ENST00000547547 plasmid transfected group or Lv-ENST00000547547 stable cells.

\section{Discussion}

Colorectal cancer (CRC) is the third most common cancer and the fourth highest cause of cancer-related deaths worldwide, with more than 1 million individuals suffering from CRC; 694,000 people died of CRC in 2012 (20). To clarify the mechanism of CRC pathogenesis, numerous protein-coding genes and non-protein-coding genes have been shown to regulate CRC initiation and development $(21,22)$. As non-coding transcripts, IncRNAs play critical roles in every aspect of cancer progression, such as initiation, invasion and migration, and could function as oncogenes or tumor suppressors $(11,23)$. In CRC, IncRNAs have been identified as regulators that widely function in CRC cell proliferation, metastasis, cell cycle progression, apoptosis and EMT (12-14). In our lncRNA microarray data, we observed that lncRNA ENST00000547547, a 434-bp transcript on human chromosome12q15 (RP11-611E13.3-001), was lower in CRC tissues.
In addition, it was also downregulated in adrenocortical carcinoma (ACC), pancreatic adenocarcinoma (PAAD), kidney chromophobe (KICH) and thyroid carcinoma (THCA) than in normal tissues as analyzed by the TCGA database data using GEPIA software (http://gepia.cancer-pku.cn/) (24). However, the role that ENST00000547547 plays in CRC development remained unknown.

In the present study, we provided the first evidence that ENST00000547547 was significantly downregulated in CRC tissues and human CRC cell lines. Furthermore, (23), the overexpression of ENST00000547547 significantly inhibited CRC cell proliferation in vitro and tumorigenesis in vivo. Although lncRNAs function widely in the developmental processes of various tumors, their precise regulatory mechanisms remain largely unknown. A previous study (25) revealed that the lncRNA Loc554202 decreased CRC cell proliferation by promoting significant arrest in the G0/G1 phase and CRC cell apoptosis. However, a study of the IncRNA PRNCR1 

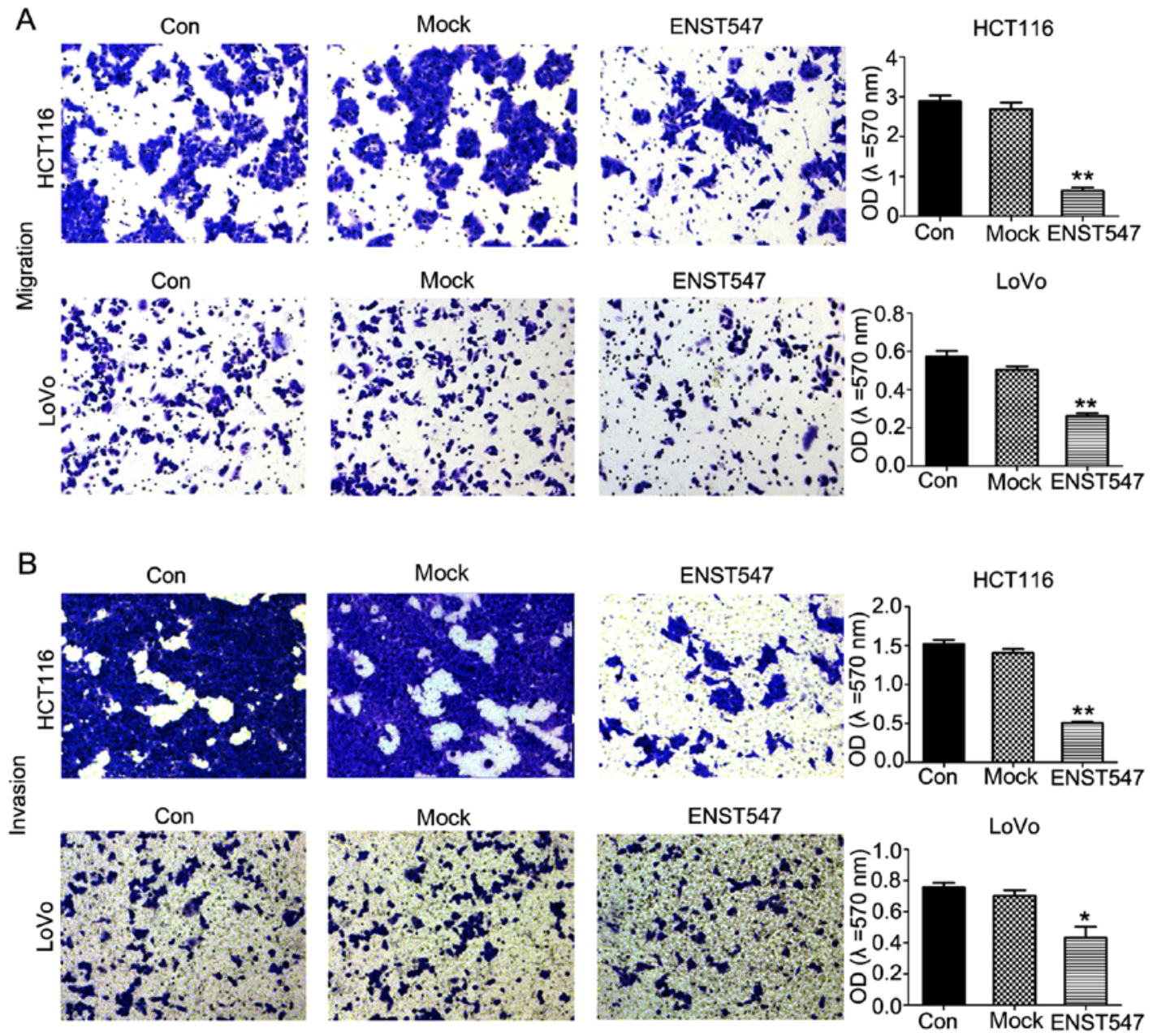

Figure 4. Effects of ENST00000547547 on CRC cell invasion and migration in vitro. (A) Transwell assays were performed in HCT116 and LoVo cells to analyze the role of ENST00000547547 in cell migration after the upregulation of ENST00000547547. (B) Transwell assays were performed to analyze the role of ENST00000547547 in the invasion ability of HCT116 and LoVo cells after the ENST00000547547 overexpression. Data represent the mean \pm SD from three independent experiments; ${ }^{*} \mathrm{P}<0.05,{ }^{* *} \mathrm{P}<0.01$. CRC, colorectal cancer; mock, pcDNA3.1 plasmid transfected group or Lv-NC stable cells; ENST547, pcDNA3.1-ENST00000547547 plasmid transfected group or Lv-ENST00000547547 stable cells.

indicated that the regulation of CRC cell proliferation was necessarily associated with affecting cell apoptosis (11). Our results revealed that the overexpression of ENST00000547547 significantly induced both cell cycle arrest in the G0/G1 phase and apoptosis in CRC cell lines. In the cell cycle progression, P21 and P27, Cip/Kip proteins, are well known for their negative role in the cell cycle $(26,27)$. In contrast, PCNA and cyclins (cyclin D1 and E1) positively regulate the cell cycle by promoting DNA replication and pushing cells from the G1 to the $S$ phase, respectively (28-30). Our results indicated that ENST00000547547 induced cell cycle arrest by upregulating the expression of P21 and P27 and downregulating the expression of cyclin D1 and E1. During apoptosis in tumor cells, the $\mathrm{Bcl}-2$ family is critical to the regulation of apoptosis, including both death antagonists such as Bcl-2 and death agonists such as Bax (31-33). Our results demonstrated that the overexpression of ENST00000547547 decreased the expression of Bcl-2 while concomitantly, increased the expression of Bax. Along with the above-mentioned study on its effects on cell cycle, these data indicated that ENST00000547547 may be another critical effector in the regulation of the cell cycle and apoptosis in CRC cells, similar to the IncRNA Loc554202.
EMT is proposed to play a key role in the acquisition of migratory or invasive capacities by cancer cells $(34,35)$. Previous studies indicated that decreased expression of E-cadherin and increased expression of $\mathrm{N}$-cadherin and vimentin are considered to be fundamental events in EMT $(36,37)$. Furthermore, ZEB1 and Sna1 can supress E-cadherin directly or indirectly, and they can be considered EMT transcription factors (38). Our results indicated that the inhibition of invasion and migration in the ENST00000547547-overexpressing CRC cells was associated with the suppression of EMT-inducing genes, indicating that ENST00000547547 may be a pleiotropic suppressor participating in EMT. And the high levels of expression of any lncRNA cause stress that may serve to induce apoptosis, such as oxidative stress-induced apoptosis (39). However, the functioning role of silencing the ENST00000547547 expression level in CRC cells was not performed, and this will be the aim of our future studies. In conclusion, the present study provided the first evidence that the IncRNA ENST00000547547 was significantly downregulated in CRC tissues and cells and that its overexpression inhibited cell proliferation, migration, invasion and EMT progression, caused cell cycle arrest 


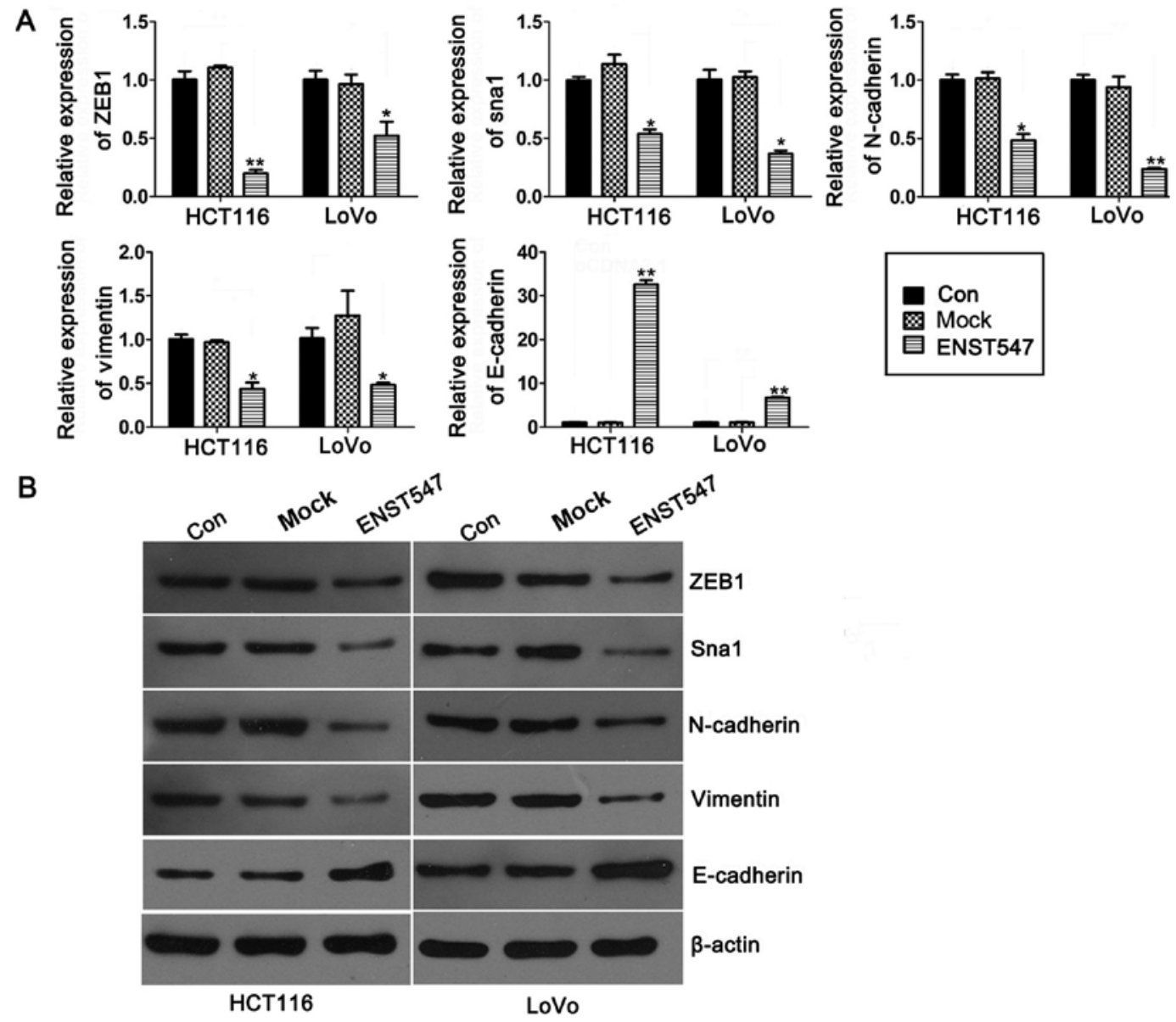

Figure 5. Effects of ENST00000547547 on epithelial-mesenchymal transition (EMT) marker gene expression. (A) The transcription levels of EMT marker genes (ZEB1, Sna1, N-cadherin, vimentin and E-cadherin) were determined by qRT-PCR analysis in the HCT116 and LoVo cells after pCDNA3.1-NC or pCDNA3.1-ENST00000547547 transfection. (B) The protein levels of EMT marker genes (ZEB1, Sna1, N-cadherin, vimentin and E-cadherin) were examined by western blot analysis in HCT116 and LoVo cells after pCDNA3.1-pCDNA3.1or pCDNA3.1-ENST00000547547 transfection. Data represent the mean \pm SD from three independent experiments; "P<0.05, ${ }^{* *} \mathrm{P}<0.01$. mock, pcDNA3.1 plasmid transfected group or Lv-NC stable cells; ENST547, pcDNA3.1-ENST00000547547 plasmid transfected group or Lv-ENST00000547547 stable cells.

at the G1 to the S phase transition in CRC cancer cells and inhibited tumorigenesis in vivo. Collectively, our study is the first to indicate that the lncRNA ENST00000547547 acts as a tumor suppressor in CRC and that it could be a new candidate biomarker for CRC prognosis and a potential therapeutic target for molecular cancer therapy.

\section{Acknowledgements}

Not applicable.

\section{Funding}

The present study was supported by the grant from the Natural Science Foundation of Hunan Province, China (grant no. 2018JJ6073), the Hunan Provincial Health and Family Planning Commission, China (no. C20180838) and the Shaoyang Science and Technology Bureau of Hunan Province, China (no. 2017GX35).

\section{Availability of data and materials}

The datasets used during the present study are available from the corresponding author upon reasonable request.

\section{Authors' contributions}

$\mathrm{XA}, \mathrm{XL}$ and $\mathrm{CL}$ conceived and designed the study. XA, QL, and XS performed the experiments. JL, GH and CC performed the experiments of the in situ hybridization (ISH) analysis and wrote the paper. JL, GX, BL, MC, WZ and HW performed the experiments of the tumor formation assay in a nude mouse model, reviewed and edited the manuscript. All authors read and approved the manuscript and agree to be accountable for all aspects of the research in ensuring that the accuracy or integrity of any part of the work are appropriately investigated and resolved.

\section{Ethics approval and consent to participate}

All experimental protocols were approved by the Shangyang Hospital of TCM(Shaoyang, China).

\section{Patient consent for publication}

Not applicable.

\section{Competing interests}

The authors state that they have no competing interests. 


\section{References}

1. Siegel R, Naishadham D and Jemal A: Cancer statistics, 2013 CA Cancer J Clin 63: 11-30, 2013.

2. Center MM, Jemal A and Ward E: International trends in colorectal cancer incidence rates. Cancer Epidemiol Biomarkers Prev 18: 1688-1694, 2009.

3. Liu S, Zheng R, Zhang M, Zhang S, Sun X and Chen W: Incidence and mortality of colorectal cancer in China, 2011. Chin J Cancer Res 27: 22-28, 2015

4. Schreuders EH, Ruco A, Rabeneck L, Schoen RE, Sung JJ Young GP and Kuipers EJ: Colorectal cancer screening: A global overview of existing programmes. Gut 64: 1637-1649, 2015.

5. Mercer TR, Dinger ME and Mattick JS: Long non-coding RNAs: Insights into functions. Nat Rev Genet 10: 155-159, 2009.

6. Kotake Y, Nakagawa T, Kitagawa K, Suzuki S, Liu N, Kitagawa M and Xiong Y: Long non-coding RNA ANRIL is required for the PRC2 recruitment to and silencing of $\mathrm{p} 15^{\mathrm{INK} 4 \mathrm{~B}}$ tumor suppressor gene. Oncogene 30: 1956-1962, 2011.

7. Li W, Zhai L, Wang H, Liu C, Zhang J, Chen W and Wei Q: Downregulation of LncRNA GAS5 causes trastuzumab resistance in breast cancer. Oncotarget 7: 27778-27786, 2016.

8. Huang J, Zhou N, Watabe K, Lu Z, Wu F, Xu M and Mo YY: Long non-coding RNA UCA1 promotes breast tumor growth by suppression of p2 $7^{\mathrm{Kip} 1}$. Cell Death Dis 5: e1008, 2014.

9. Zhao Y, Guo Q, Chen J, Hu J, Wang S and Sun Y: Role of long non-coding RNA HULC in cell proliferation, apoptosis and tumor metastasis of gastric cancer: A clinical and in vitro investigation. Oncol Rep 31: 358-364, 2014.

10. Chen X, Liu B, Yang R, Guo Y, Li F, Wang L and Hu H: Integrated analysis of long non-coding RNAs in human colorectal cancer. Oncotarget 7: 23897-23908, 2016.

11. Yang L, Qiu M, Xu Y, Wang J, Zheng Y, Li M, Xu L and Yin R: Upregulation of long non-coding RNA PRNCR1 in colorectal cancer promotes cell proliferation and cell cycle progression. Oncol Rep 35: 318-324, 2016.

12. Lai MC, Yang Z, Zhou L, Zhu QQ, Xie HY, Zhang F, Wu LM, Chen LM and Zheng SS: Long non-coding RNA MALAT-1 overexpression predicts tumor recurrence of hepatocellular carcinoma after liver transplantation. Med Oncol 29: 1810-1816, 2012.

13. Yang XD, Xu HT, Xu XH, Ru G, Liu W, Zhu JJ, Wu YY, Zhao K, Wu Y, Xing CG, et al: Knockdown of long non-coding RNA HOTAIR inhibits proliferation and invasiveness and improves radiosensitivity in colorectal cancer. Oncol Rep 35: 479-487, 2016.

14. Niu H, Hu Z, Liu H, Hu G, Yang B, Wu S and Li F: Long non-coding RNA AK027294 involves in the process of proliferation, migration, and apoptosis of colorectal cancer cells. Tumor Biol 37: 10097-10105, 2016.

15. Li J, Li X, Cen C, Ai X, Lin C and Hu G: The long non-coding RNA ENST00000547547 reduces 5-fluorouracil resistance of colorectal cancer cells via competitive binding to microRNA-31. Oncol Rep 39: 217-226, 2018

16. Li H, Wang X, Wen C, Huo Z, Wang W, Zhan Q, Cheng D, Chen H, Deng X, Peng C, et al: Long noncoding RNA NORAD, a novel competing endogenous RNA, enhances the hypoxia-induced epithelial-mesenchymal transition to promote metastasis in pancreatic cancer. Mol Cancer 16: 169, 2017.

17. Portt L, Norman G, Clapp C, Greenwood M and Greenwood MT: Anti-apoptosis and cell survival: A review. Biochim Biophys Acta 1813: 238-259, 2011.

18. Punchihewa C, Inoue A, Hishiki A, Fujikawa Y, Connelly M, Evison B, Shao Y, Heath R, Kuraoka I, Rodrigues P, et al: Identification of small molecule proliferating cell nuclear antigen (PCNA) inhibitor that disrupts interactions with PIP-box proteins and inhibits DNA replication. J Biol Chem 287: 14289-14300, 2012.

19. Wang SC: PCNA: A silent housekeeper or a potential therapeutic target? Trends Pharmacol Sci 35: 178-186, 2014.
20. Ferlay J, Soerjomataram I, Dikshit R, Eser S, Mathers C, Rebelo M, Parkin DM, Forman D and Bray F: Cancer incidence and mortality worldwide: Sources, methods and major patterns in GLOBOCAN 2012. Int J Cancer 136: E359-E386, 2015.

21. Punt CJ, Koopman M and Vermeulen L: From tumour heterogeneity to advances in precision treatment of colorectal cancer. Nat Rev Clin Oncol 14: 235-246, 2017.

22. Wu WK, Law PT, Lee CW, Cho CH, Fan D, Wu K, Yu J and Sung JJ: MicroRNA in colorectal cancer: From benchtop to bedside. Carcinogenesis 32: 247-253, 2011.

23. Long Y, Wang X, Youmans DT and Cech TR: How do lncRNAs regulate transcription? Sci Adv 3: eaao2110, 2017.

24. Tang Z, Li C, Kang B, Gao G, Li C and Zhang Z: GEPIA: A web server for cancer and normal gene expression profiling and interactive analyses. Nucleic Acids Res 45: W98-W102, 2017.

25. Ding J, Lu B, Wang J, Wang J, Shi Y, Lian Y, Zhu Y, Wang J, Fan Y, Wang Z, et al: Long non-coding RNA Loc554202 induces apoptosis in colorectal cancer cells via the caspase cleavage cascades. J Exp Clin Cancer Res 34: 100, 2015.

26. Coqueret O: New roles for p21 and p27 cell-cycle inhibitors: A function for each cell compartment? Trends Cell Biol 13: 65-70, 2003.

27. Liu Y, Jing Z, Zhang W, Gan J, Hu C, Huang G and Zhang Y: lncRNA GAS5 enhances G1 cell cycle arrest via binding to YBX1 to regulate p21 expression in stomach cancer. Sci Rep 5: 10159, 2015.

28. Cayrol C, Knibiehler M and Ducommun B: p21 binding to PCNA causes G1 and G2 cell cycle arrest in p53-deficient cells. Oncogene 16: 311-320, 1998.

29. Liu L, Zhang H, Shi L, Zhang W, Yuan J, Chen X, Liu J, Zhang Y and Wang Z: Inhibition of Racl activity induces G1/S phase arrest through the GSK3/cyclin D1 pathway in human cancer cells. Oncol Rep 32: 1395-1400, 2014.

30. Rosenberg E, Demopoulos RI, Zeleniuch-Jacquotte A, Yee H, Sorich J, Speyer JL and Newcomb EW: Expression of cell cycle regulators $\mathrm{p} 57^{\mathrm{KIP} 2}$, cyclin D1, and cyclin E in epithelial ovarian tumors and survival. Hum Pathol 32: 808-813, 2001.

31. Wang JL, Liu D, Zhang ZJ, Shan S, Han X, Srinivasula SM, Croce CM, Alnemri ES and Huang Z: Structure-based discovery of an organic compound that binds Bcl-2 protein and induces apoptosis of tumor cells. Proc Natl Acad Sci USA 97: 7124-7129, 2000.

32. Yip KW and Reed JC: Bcl-2 family proteins and cancer. Oncogene 27: 6398-6406, 2008.

33. Koda M, Przystupa W, Jarzabek K, Wincewicz A, Kanczuga-Koda L, Tomaszewski J, Sulkowska M, Wolczynski S and Sulkowski S: Expression of insulin-like growth factor-I receptor, estrogen receptor alpha, Bcl-2 and Bax proteins in human breast cancer. Oncol Rep 14: 93-98, 2005.

34. Kramer N, Walzl A, Unger C, Rosner M, Krupitza G, Hengstschläger $\mathrm{M}$ and Dolznig $\mathrm{H}$ : In vitro cell migration and invasion assays. Mutat Res 752: 10-24, 2013.

35. Singh A and Settleman J: EMT, cancer stem cells and drug resistance: An emerging axis of evil in the war on cancer. Oncogene 29: 4741-4751, 2010.

36. Li L and Li W: Epithelial-mesenchymal transition in human cancer: Comprehensive reprogramming of metabolism, epigenetics, and differentiation. Pharmacol Ther 150: 33-46, 2015.

37. Nalla AK, Estes N, Patel J and Rao JS: N-cadherin mediates angiogenesis by regulating monocyte chemoattractant protein-1 expression via PI3K/Akt signaling in prostate cancer cells. Exp Cell Res 317: 2512-2521, 2011.

38. Peinado H, Olmeda D and Cano A: Snail, Zeb and bHLH factors in tumour progression: An alliance against the epithelial phenotype? Nat Rev Cancer 7: 415-428, 2007.

39. Zhang D, Lee H, Haspel JA and Jin Y: Long noncoding RNA FOXD3-AS1 regulates oxidative stress-induced apoptosis via sponging microRNA-150. FASEB J 31: 4472-4481, 2017. 